\title{
China needs a national intelligent syndromic surveillance system
}

To the editor - An epidemic can open a window of opportunity for reflection on and a revolution in disease control and prevention. In China, the 2003 severe acute respiratory syndrome (SARS) epidemic urged the national paper-based disease-reporting system to be transformed into the national notifiable disease reporting system (NNDRS), a system operated by the Chinese Center for Disease Control and Prevention (CDC) to facilitate the complete and timely reporting of cases of infectious disease. The coronavirus disease 2019 (COVID-19) pandemic is now posing an urgent call for upgrading the NNDRS further.

With the paper-based disease-reporting system that had been used in China before 2004, district-level (or county-level) aggregated cases were reported laboriously by mail through the municipal (or prefectural) CDC, the provincial CDC and, finally, the national CDC. Because of the SARS outbreak, the operational mechanism of the paper-based system had been updated for more responsiveness, toward the web-based NNDRS, with which individual cases from every hospital, township and upper-level primary healthcare clinic are reported directly to the national CDC. Before the outbreak of COVID-19, the NNDRS had been monitoring the progress of 39 infectious diseases, identified in the Law on the Prevention and Control of Infectious Diseases of China.

The average time between the identification of a disease case and reporting of that case to the Chinese CDC has been shortened from 29 days to under 2 hours for all Category A and specified Category $B$ infectious diseases, or 24 hours for all unspecified Category B infectious diseases. On 20 January 2020, COVID-19 was officially defined as a Category B infectious disease but was reported as a Category A infectious disease within 2 hours. Nevertheless, it may still take time for different levels of the CDC to confirm the suspected cases.

Spatial life course epidemiology $y^{1}$ the study of evolving agents, mobile hosts and changing environments as a dynamic epidemiological triad ${ }^{2}$ - could upgrade the current NNDRS into a national intelligent syndromic surveillance system (NISSS), with which early risk could be identified on the basis of information about patients' symptoms (e.g., cough, fever, diarrhea, vomiting and shortness of breath) during

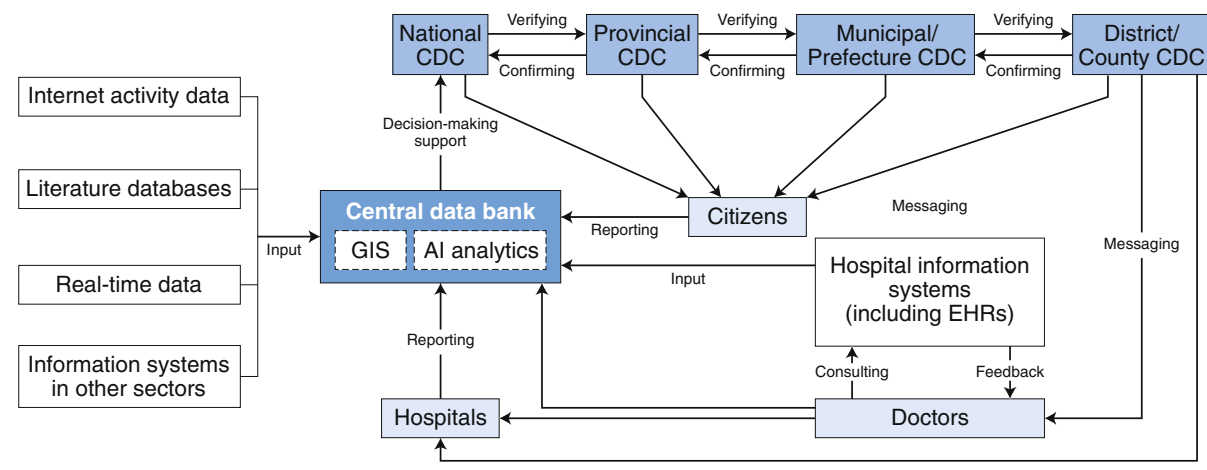

Fig. 1 | The system architecture of the NISSS in China. Al, artificial intelligence; EHR, electronic health record; GIS, geographic information system.

the early phases of illness, sometimes with the assistance of surrogate data that indicate early illness (e.g., school or work absenteeism data, or veterinary data such as unexpected avian deaths) ${ }^{3}$. Going beyond traditional epidemiology, spatial life course epidemiology utilizes an array of advanced technologies, including artificial intelligence, to support both data analysis and decision making and to automate the analysis of risk factors, transmission patterns and potential measures that could be used to control the spread of the disease, which could dramatically improve the public health response $e^{4,5}$.

For this system to work at its best capacity, information input from end users, such as from doctors, hospital information systems, citizens, medical literature databases and internet activities, and other external sources is required and enabled by the current technology (Fig. 1). The NISSS could also field duplicate reporting and reporting inconsistency from different sources.

The protection of individual privacy rights should be paramount in the NISSS, which can be fully realized in the era of technology. A careful protocol must thus be discussed thoroughly among multiple information providers; this should include solving several key issues, such as the following: suspected cases or places reported and/or positioned by individuals (e.g., doctors or citizens) should be made anonymous and should be available only to certain groups before confirmation; individuals posting suspected cases or places should be made anonymous and should be identifiable only by certain groups; actual locations of confirmed cases may be made anonymous and/or offset toward a random direction by a certain distance before being shown in the NISSS; and the information on confirmed cases should be, to different degrees, available to various groups ${ }^{6}$.

The CDC will be made more intelligent in detecting early risk so that three key lessons from the SARS outbreak could be better taken: honesty is needed; controversy can lead to lost chances; and conclusions may be premature. ${ }^{7}$ The NISSS will enable coordinated data-sharing mechanisms ahead of an outbreak, improving the reporting, quality and accessibility of data in an era of high-impact and high-frequency epidemics ${ }^{8}$.

\section{Peng Jia (D) 1,2,4凶 and Shujuan Yang 2,3,4凶} ${ }^{1}$ Department of Land Surveying and Geo-Informatics, The Hong Kong Polytechnic University, Hong Kong, China. ${ }^{2}$ International Initiative on Spatial Lifecourse Epidemiology, Hong Kong, China. ${ }^{3}$ West China School of Public Health and West China Fourth Hospital, Sichuan University, Chengdu, China. ${ }^{4}$ These authors contributed equally: Peng Jia and Shujuan Yang.

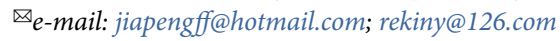

Published online: 20 May 2020

https://doi.org/10.1038/s41591-020-0921-5

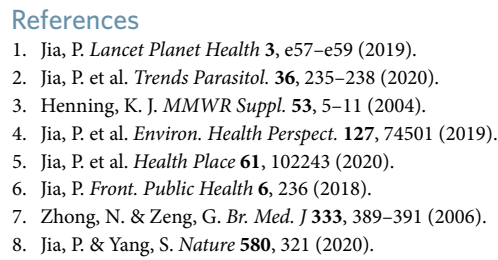

The authors declare no competing interests. 